\title{
SOME PROBLEMS CONCERNING BASES IN BANACH SPACES
}

\author{
J. R. HOLUB ${ }^{1}$
}

1. Introduction. A basis $\left(x_{i}, f_{i}\right)$ for a Banach space $X$ having the property that $0<\inf _{i}\left\|x_{i}\right\| \leqq \sup _{i}\left\|x_{i}\right\|<+\infty$ is said to be

(1) of type $P$ [4] provided $\sup _{n}\left\|\sum_{i=1}^{n} x_{i}\right\|<+\infty$,

(2) of type $P^{*}[4]$ provided $\sup _{n}\left\|\sum_{i=1}^{n} f_{i}\right\|<+\infty$,

(3) of type swc $_{0}[2]$ if the sequence $\left\{x_{n}\right\}$ has a subsequence $\left\{x_{n_{i}}\right\}$ weakly convergent to zero.

(4) of type $\left(\mathrm{swc}_{0}\right)^{*}[2]$ if the sequence $\left\{f_{n}\right\}$ in $X^{*}$ is of type $\mathrm{swc}_{0}$. Foias and Singer [2] have asked the following questions:

(Q1) If $\left(x_{i}\right)$ is a basis for a Banach space $X$ such that 0 is not a weak limit point of the set $\left\{x_{i}\right\}$, must there exist an $f$ in $X^{*}$ for which $\left|f\left(x_{i}\right)\right|$ $\geqq 1$ for all $i$ ?

(Q2) Does $L^{1}[0,1]$ have a basis of type P?

(Q3) Does $L^{1}[0,1]$ have a basis of type swc $_{0}$ ?

(Q4) Does $C[0,1]$ have a basis of type $\left(\mathrm{swc}_{0}\right) *$ ?

The purpose of this paper is to give a negative answer to (Q1) and affirmative answers to (Q2)-(Q4).

2. The question (Q1). We will need the following notion of "block perturbation basis" introduced by Pełczyński and Singer [3].

(2.1) Definition [3]. Let $\left(w_{i}\right)$ be a basis for a Banach space $E$ such that $\inf _{i}\left\|w_{i}\right\|>0$. A block perturbation of $\left(w_{i}\right)$ is any sequence $\left(V_{k}\right)$ in $E$ of the form

$$
\begin{aligned}
V_{k} & =w_{k} \text { for } k \neq p_{n} \\
& =w_{p_{n}}+u_{n} \text { for } k=p_{n}(n=1,2, \cdots),
\end{aligned}
$$

where $u_{n}=\sum_{i=m_{n-1}+1}^{p_{n}-1} a_{i} w_{i}+\sum_{i=p_{n}+1}^{m_{n}} a_{i} w_{i}$ with $\left\|u_{n}\right\| \leqq M$ for $n=1,2$, $\cdots,\left\{m_{n}\right\}$ and $\left\{p_{n}\right\}$ are increasing sequences of positive integers such that $m_{0}=0, m_{n-1}+1 \leqq p_{n} \leqq m_{n}$, and $\left(a_{i}\right)$ is a sequence of scalars.

The following result is due to Pełczyński and Singer [3].

(2.2) THEOREM. If $\left(w_{i}\right)$ is a basis for $E$ such that inf $_{i}\left\|w_{i}\right\|>0$, then every block perturbation $\left(V_{k}\right)$ of $\left(w_{i}\right)$ is a basis for $E$.

Received by the editors March 25, 1969 and, in revised form, May 12, 1969.

1 This paper is part of the author's doctoral research currently in progress at Louisiana State University under the direction of Professor J. R. Retherford. The author wishes to thank Professor Retherford for his helpful suggestions regarding this paper. 
Let $c$ denote the space of all convergent sequences with the sup norm. It is well known that $c$ is isomorphic to the space $C(\hat{I})$ (where $\hat{I}$ denotes the one point compactification of the positive integers) and that the sequence $x_{1}=(1,1,1,1, \cdots), x_{2}=(0,1,1,1, \cdots), x_{3}$ $=(0,0,1,1,1, \cdots), \cdots$, is a basis for $c$.

It follows that the dual space of $c$ may be identified with those sequences $\mu=\left(\mu_{1}, \mu_{2}, \cdots, \mu_{\infty}\right)$ for which $\sum_{i}\left|\mu_{i}\right|+\left|\mu_{\infty}\right|<\infty$ (we denote by $\mu_{\infty}$ the $\mu$-measure of the ideal element of $\left.\hat{I}\right)$. If $\mu \in c^{*}$ and $a=\left(a_{i}\right) \in c$, then $\mu(a)=\sum_{i} \mu_{i} a_{i}+\mu_{\infty} \cdot \lim _{i} a_{i}$. In particular, $\mu\left(x_{n}\right)$ $=\sum_{i \geqq n} \mu_{i}+\mu_{\infty}$.

It is easy to see that the sequence $\left(y_{i}\right)$ defined by

$$
\begin{aligned}
& y_{1}=\left(x_{1}, 0\right), y_{2}=\left(0, x_{1}\right), y_{3}=\left(0, x_{2}\right), y_{4}=\left(x_{2}, 0\right), y_{5}=\left(x_{3}, 0\right), \\
& y_{6}=\left(0, x_{3}\right), y_{7}=\left(0, x_{4}\right), \cdots, \text { is a basis for } c \oplus c .
\end{aligned}
$$

Let $\left(a_{n}\right)$ be any dense sequence in $[-1,1]$. Applying Theorem (2.2), we see that the sequence $\left(z_{i}\right)$ defined by

$$
\begin{aligned}
& z_{1}=\left(x_{1}, 0\right), z_{2}=\left(a_{1} x_{1}, x_{1}\right), z_{3}=\left(0, x_{2}\right), z_{4}=\left(x_{2}, a_{1} x_{1}\right), \\
& z_{5}=\left(x_{3}, 0\right), z_{6}=\left(a_{2} x_{3}, x_{3}\right), z_{7}=\left(0, x_{4}\right), z_{8}=\left(x_{4}, a_{2} x_{4}\right), \\
& z_{9}=\left(x_{5}, 0\right), z_{10}=\left(a_{3} x_{5}, x_{5}\right), \cdots \text { is a basis for } c \oplus c .
\end{aligned}
$$

Moreover, if $\mu_{0}=(0,0, \cdots, 1) \in c^{*}$, then $f=\left(\mu_{0}, 0\right)$ and $g=\left(0, \mu_{0}\right)$ are elements of $(c \oplus c)^{*}$. Clearly, no basis element $z_{i}$ is contained in the weak 0 -neighborhood $U\left[0 ; f, g ; \frac{1}{2}\right]$.

However, if $(\mu, \lambda) \in(c \oplus c)^{*}$, then

$$
\liminf _{n \rightarrow \infty}\left|(\mu, \lambda)\left[z_{4 n+2}\right]\right|=\liminf \left|a_{n} \mu_{\infty}+\lambda_{\infty}\right|
$$

and

$$
\liminf _{n \rightarrow \infty}\left|(\mu, \lambda)\left[z_{4_{n}}\right]\right|=\liminf \left|\mu_{\infty}+a_{n} \lambda_{\infty}\right| .
$$

Since the sequence $\left(a_{n}\right)$ is dense in $[-1,1]$, one of these two numbers is zero (for each fixed $(\mu, \lambda)$ ).

Thus 0 is not a weak limit point of the basis $\left(z_{i}\right)$ and there does not exist an $f \in(c \oplus c)^{*}$ for which $\left|f\left(z_{i}\right)\right| \geqq 1$ for all $i$. Therefore (Q1) is answered negatively.

Note. Since $c \oplus c=c_{0} \oplus c_{0}=c_{0}$, the basis constructed in this example may be chosen in the "nice" space $c_{0}$. The " $=$ " here meaning "is linear homeomorphic to."

3. The question $(Q 2)$. Using the "perturbation theorem" (2.2), we will construct a basis $\left(x_{i}, x_{i}^{*}\right)$ having the property that $\left(x_{i}\right)$ is of type 
$P$ and $\left(x_{i}^{*}\right)$ is similar to a basis for $L^{1}[0,1]$. It then follows that $L^{1}[0,1]$ has a basis of type $P^{*}[4]$, and hence a basis of type $P[4]$. Let $\left(z_{i}\right)_{i=1}^{\infty}$ denote the normalized Haar basis for $L^{1}[0,1]$. By definition $z_{1}(t) \equiv 1$ and,

$$
\begin{aligned}
z_{2} k_{+l}(t)=2^{k} \quad \text { for } t \in\left(\frac{2 l-2}{2^{k+1}}, \frac{2 l-1}{2^{k+1}}\right) \\
=-2^{k} \quad \text { for } t \in\left(\frac{2 l-1}{2^{k+1}}, \frac{2 l}{2^{k+1}}\right) \\
=0 \quad \text { for other } t \\
\quad\left(l=1,2, \cdots, 2^{k} ; k=0,1,2, \cdots\right) .
\end{aligned}
$$

It follows easily from the orthonormality of the Haar functions that the sequence of coefficient functionals $\left(z_{i}^{*}\right)$ in $L^{\infty}[0,1]$ associated with the basis $\left(z_{i}\right)$ is defined by

$$
\begin{aligned}
z_{1}^{*} & =z_{1}, \\
z_{2^{k}+l}^{*} & =\frac{1}{2^{k}} z_{2^{k}+l .}
\end{aligned}
$$

We will perturb the basis $\left(z_{i}^{*}\right)$ for $\left[z_{i}^{*}\right]$ according to (2.2) to get another basis $\left(x_{i}\right)$ for $\left[z_{i}^{*}\right]$ which is of type $P$ and whose coefficient functionals $\left(x_{i}^{*}\right)$ have the same closed linear span as those of the basis $\left(z_{i}^{*}\right)$, i.e. $\left[x_{i}^{*}\right]=L^{1}[0,1]$.

The elements of the basis $\left(z_{i}^{*}\right)$ to be perturbed are those of the form $z_{2^{m}-1}^{*}$, for $m=3,4, \cdots$. Since $\left\|z_{i}^{*}\right\|=1$ for all $i$, and $\left\|\sum_{j=2^{m-1}}^{2^{m}-2} z_{j}^{*}\right\|=1$ for all $m \geqq 3$ by definition of the functions $\left(z_{j}^{*}\right)$, the theorem (2.2) shows that the sequence $\left(x_{i}\right)$ in $\left[z_{i}{ }^{*}\right]$ defined by

$$
\begin{aligned}
x_{i} & =z_{i}^{*} \text { for } i \neq 2^{m}-1, m \geqq 3, \\
& =z_{2^{m}-1}^{*}-\sum_{j=2^{m-1}}^{2^{m}-2} z_{j}^{*} \text { for } i=2^{m}-1, m \geqq 3,
\end{aligned}
$$

is a basis for $\left[z_{i}{ }^{*}\right]$. Moreover, if $\left(x_{i}{ }^{*}\right)$ denotes the sequence of coefficient functionals associated with the basis $\left(x_{i}\right)$, then an examination of the proof of (2.2) [3] shows that $\left[x_{i}^{*}\right]$ is identical to the closed linear span of the sequence of coefficient functionals associated with $\left(z_{i}^{*}\right)$. Since this last is just $\left[z_{i}\right]=L^{1}[0,1]$, we have that $\left[x_{i}^{*}\right]=L^{1}[0,1]$.

We claim that $\left(x_{i}\right)$ is of type $P$ in $\left[z_{i}^{*}\right]$. In fact, $\left\|x_{i}\right\|=1$ for all $i$, and for any $n=2^{m}+l$, where $1 \leqq l \leqq 2^{m}-2$, 


$$
\begin{aligned}
&\left\|\sum_{i=1}^{n} x_{i}\right\| \leqq \\
&\left\|\sum_{i=1}^{3} x_{i}\right\|+\left\|\sum_{i=4}^{2^{m}-1} x_{i}+\right\| \sum_{i=2^{m}}^{2^{m}+l} x_{i} \| \\
&=\left\|\sum_{i=1}^{3} x_{i}+\right\| \sum_{j=3}^{m} z_{2^{j}-1}^{*}\left\|+\mid \sum_{i=2^{m}}^{2^{m}+l} z_{i}^{*}\right\|
\end{aligned}
$$

by definition of the sequence $\left(x_{i}\right)$. If $l=2^{m}-1$ the above becomes

$$
\left\|\sum_{i=1}^{n} x_{i}\right\| \leqq\left\|\sum_{i=1}^{3} x_{i}\right\|+\left\|\sum_{j=3}^{m+1} z_{2}{ }^{j}-1\right\|
$$

while if $l=2^{m}$, we have

$$
\left\|\sum_{i=1}^{n} x_{i}\right\| \leqq\left\|\sum_{i=1}^{3} x_{i}\right\|+\left\|\sum_{j=3}^{m+1} z_{2}^{*}\right\|+\left\|z_{2}{ }^{m+1}\right\| .
$$

In any case, by definition of the Haar functions, $\left\|\sum_{j=3}^{p} z_{2^{j}-1}^{*}\right\|=1$ for all $p \geqq 3$, and $\left\|\sum_{i=2^{m}}^{2^{m}+l} z_{i}^{*}\right\|=1$ for all $1 \leqq l \leqq 2^{m}-2$. Hence we see that $\left(x_{i}\right)$ is of type $P$. It follows that $\left(x_{i}^{*}\right)$ is similar to a basis of type $P^{*}$ for $L^{1}[0,1]$, and according to our previous remarks, $L^{1}[0,1]$ has a basis of type $P$.

REMARK 1 . Since a basis of type $P^{*}$ is certainly weakly closed [2], it follows from the above that $L^{1}[0,1]$ has a weakly closed basis. Also, since the coefficient functionals of the basis of type $P$ for $L^{1}[0,1]$ are of type $P^{*}$, it follows that $L^{1}[0,1]$ has a (weakly closed) ${ }^{*}$ basis [2].

These observations answer two other questions raised by Foias and Singer [2].

REMARK 2. The method of perturbation which was applied above to obtain a type $P$ basis for $\left[z_{i}^{*}\right]$ will clearly also yield a type $P$ basis for $C[0,1]$ when applied to the usual Schauder basis for $C[0,1]$. Foias and Singer have previously shown, using a different construction, that $C[0,1]$ has a basis of type $P[2]$.

4. The questions $(\mathrm{Q} 3)$ and $(\mathrm{Q} 4)$. Zippin has proved the following lemma.

(4.1) Lemma (ZIPPIN [5]). Let $\left(x_{i}\right)_{i=1}^{\infty}$ be a basis for a Banach space $X$. Assume that $y_{k}=\sum_{i=p_{k}+1}^{p_{k+1}} a_{i} x_{i} \neq 0$, where $\left\{p_{k}\right\}_{k=2}^{\infty}$ is an increasing sequence of positive integers and $p_{1}=0$. Then there exists a basis $\left(z_{i}\right)_{i=1}^{\infty}$ for $X$ such that for each $k \geqq 1, z_{p_{k+1}}=y_{k}$.

Moreover, as Zippin's proof shows, if $\left(z_{i}^{*}\right)$ is the sequence of coefficient functionals associated with the basis $\left(z_{i}\right)$, then $\left[z_{i}^{*}\right]=\left[x_{i}^{*}\right]$, 
where $\left(x_{i}^{*}\right)$ is the sequence of coefficient functionals associated with $\left(x_{i}\right)$.

(4.2) Proposition. Let $X$ be a Banach space with a basis in which weak and norm convergence are not equivalent. Then $X$ has a basis of type $\mathrm{swc}_{0}$.

Proof. Since weak and norm convergence are not equivalent there exists a sequence $\left(w_{i}\right)$ in $X$ which is weakly convergent to zero but for which $\inf _{i}\left\|w_{i}\right\|>0$.

Let $\left(x_{i}\right)$ be a basis for $X$. By a theorem of Bessaga and Pełczyński [1] there is a subsequence $\left(w_{n_{k}}\right)$ which is a basic sequence in $X$ similar to a block basic sequence $\left(y_{k}\right)_{k=1}^{\infty}=\left(\sum_{i=p_{k}+1}^{p_{k+1}} a_{i} x_{i}\right)_{k=1}^{\infty}$, where $p_{1}=0$ and $\left(p_{i}\right)_{i=2}^{\infty}$ is an increasing sequence of positive integers. It follows that $\left(y_{k}\right)$ is weakly convergent to zero.

By Zippin's Lemma (4.1) there exists a basis $\left(z_{i}\right)$ for $X$ such that $z_{p_{k+1}}=y_{k}$. Since $\left(y_{k}\right)$ converges weakly to zero, the basis $\left(z_{i}\right)$ is clearly of type swc $_{0}$.

The following corollary to (4.1) answers (Q3).

(4.3) Corollary. The space $L^{1}[0,1]$ has a basis of type $\mathrm{swc}_{0}$.

Proof. The sequence $\{\sin 2 n \pi x\}$ is weakly convergent to zero in $L^{1}[0,1]$ but not norm convergent. Apply Proposition (4.2).

To answer $(Q 4)$, let $\left(x_{i}, x_{i}^{*}\right)$ be any basis for $L^{1}[0,1]$ for which $\left[x_{i}^{*}\right]$ is isomorphic to $C[0,1]$ (e.g. let $\left(x_{i}\right)$ be the normalized Haar basis for $\left.L^{1}[0,1][2]\right)$. By the above construction there exists a basis $\left(z_{i}\right)$ for $L^{1}[0,1]$ which is of type $\mathrm{swc}_{0}$ and whose coefficient functionals $\left(z_{i}^{*}\right)$ span a space isomorphic to $\left[x_{i}^{*}\right]=C[0,1]$. Therefore, since the sequence of coefficient functionals associated with $\left(z_{i}^{*}\right)$ is similar to $\left(z_{i}\right)$ we see that $C[0,1]$ has a basis of type $\left(\mathrm{swc}_{0}\right)^{*}$, and $\left(\mathrm{Q}^{4}\right)$ is also answered affirmatively.

\section{BIBLIOGRAPHY}

1. C. Bessaga and A. Pełczyński, On bases and unconditional convergence of series in Banach spaces, Studia Math. 17 (1958), 151-164.

2. C. Foias and I. Singer, On bases in $C[0,1]$ and $L^{1}[0,1]$, Rev. Roumaine Math. Pures Appl. 10 (1965), 931-960.

3. A. Pełczynski and I. Singer, On non-equivalent bases and unconditional bases in Banach spaces, Studia Math. 25 (1964), 5-25.

4. I. Singer, Bases, basic sequences and reflexivity of Banach spaces, Studia Math. 21 (1962), 351-369.

5. M. Zippin, $A$ remark on bases and reflexivity in Banach spaces, Israel J. Math. 6 (1968), 74-79.

The Louisiana State University 